\title{
$\mathrm{R}_{\text {evista }} \mathrm{B}_{\text {rasileira de }} \mathrm{F}_{\text {isica }} \mathrm{T}_{\text {ecnológica }} \mathrm{A}_{\text {plicada, v.2, n.2, dezembro de } 2015 .}$
}

\section{¿Como flotan los submarinos?}

\section{Como flutuam os submarinos?}

\section{As the submarines float?}

Juan Carlos Sánchez Reyes ${ }^{1}$; María José Torres Fernández; Adriana Carolina Vasquez Ramirez.

Resumen: El proceso mediante el cual flotan los cuerpos obedece a leyes físicas muy bien establecidas, pero al tratarlas de manera independiente parecieran entrar en conflictos y se contradicen. El fenómeno de la flotabilidad de un submarino nos permite integrar y convalidar todos los principios y leyes de la física hidrostática de una manera muy formal, sustentada con experiencias alternativas y de fácil elaboración en el aula de clase y con materiales de bajo costo. Este artículo ofrece experimentos hidrostáticos simples con el fin de responder a las preguntas y conceptos a los estudiantes. Los resultados se produjeron a partir de la utilización de estos experimentos con estudiantes de Licenciatura en Ciencias Físicas.

Palabras clave: fluidos; hidrostática; submarino; enseñanza de Física.

Resumo: O processo pelo qual os corpos flutuam obedecem a leis físicas muito bem estabelecidas, porém, ao tratar essas leis de forma independente, parecem entrar em conflitos e contradições. $\mathrm{O}$ fenómeno da flutuabilidade de um submarino nos permite integrar e convalidar todos os principios e leis da física hidrostática de uma maneira formal, sustentada com experiências alternativas e de fácil elaboração para trabalhar em sala de aula e com materias de baixo custo. Este artigo apresenta três experimentos simples de hidrostática com o objetivo de esclarecer dúvidas e conceitos aos alunos. A análise dos resultados se deu a partir do uso desses experimentos com alunos de Licenciatura em Ciências Física.

Palavras-chave: fluidos; hidrostática; submarino ; ensino de Física

\begin{abstract}
The process by which floating bodies due to well-established physical laws, but to treat them independently enter into conflicts and seem contradictory. The phenomenon of buoyancy of a submarine allows us to integrate and validate all the principles and laws of physics hydrostatic a very formal way, supported with easy to prepare alternatives and experiences in the classroom and with inexpensive materials. This article offers simple hydrostatic experiments in order to answer questions and concepts to students. The results occurred from the use of these experiments with students of Graduate in Physical Sciences.
\end{abstract}

Key-words: Fluids; Hydrostatic; Submarine; Physical Teaching. 


\section{Introducción}

Desde muy corta edad los chicos exhiben una curiosidad innata por los fenómenos naturales. En ese proceso ellos formulan preguntas y proponen modelos que los someten a prueba en la medida que juegan y aprenden. Todo ese aprendizaje formará parte de sus vivencias y poco a poco cobrará significado en su quehacer diario.

Para evitar la aversión en el estudio de las ciencias y en particular de la física, se debe evitar el aprendizaje mecánico ya que dificultará la consolidación de los conceptos y el razonamiento lógico abstracto. Por otro lado, una vez estructurado el conocimiento del tema, el estudiante confronta lo aprendido con distintas situaciones que en determinadas situaciones no satisfacen sus expectativas y entran en conflictos. El presente trabajo intentará recrear estas situaciones y propondrá experimentos que permitirán recrear y aclarar estas situaciones.

\section{Teoria de aprendizage}

Para los profesores de ciencias, el propósito de la actividad docente, entre otras cosas, es contribuir a la motivación de los estudiantes, a explorar una u otras variantes en el planteamiento de un problema o fenómeno natural empleando el método científico, con el propósito de mostrarles algún procedimiento, técnica o la concordancia entre la teoría y la práctica. Al final se espera reforzar la teoría y adquirir destrezas prácticas, manejo del instrumental y la aplicación del método científico. El tema tratado en este artículo: "La flotabilidad variable de los cuerpos" se presta para diversas interpretaciones tal como se ha evidenciado en el aula de clase. Dichas interpretaciones tienden a ser divergentes y contradictorias; los estudiantes afirman que los cuerpos livianos flotan mientras que los pesados se hunden, pero ¿un submarino es pesado o liviano?, -dado que un submarino a veces flota y otras veces se hunde, ¿cambia su peso?

Las respuestas recogidas en el aula de clase parece ser contradictorias. El cambio conceptual que produce en los estudiantes una situación contradictoria, entre lo que ellos saben (conocimientos previos) y los conocimientos nuevos, provoca un desequilibrio cognitivo que conduce a un nuevo conocimiento más amplio y ajustado a la realidad y sigue enriqueciéndose en nuevos procesos de aprendizaje a través de ciclos evolutivos. 
Las propuestas pedagógica en la que se fundamenta el siguiente trabajo y que se consolida con lo anteriormente expuesto se le conoce como Conflicto Cognitivo de Piaget (1975).

\section{Fluidos}

En el campo de la física, uno de los temas que ha interesado al hombre desde la antigüedad ha sido la hidráulica por sus múltiples aplicaciones. Desde los antiguos egipcios, griegos y romanos, los grandes avances de la civilización giraban en torno al manejo de las aguas que jugó un papel fundamental en el asentamiento y desarrollo de las civilizaciones.

La hidráulica es una rama de la física y la ingeniería que se encarga del estudio de las propiedades mecánicas de los fluidos. Todo esto depende de las fuerzas que se interponen con la masa (fuerza) y empuje de la misma (LEA y BURKE; 1999, p. 434).

Pero, ¿que son fluidos? Se denomina fluido a un tipo de medio continuo formado por alguna sustancia entre cuyas moléculas hay una fuerza de atracción débil. Los fluidos se caracterizan por cambiar de forma sin que existan fuerzas restitutivas tendentes a recuperar la forma original (lo cual constituye la principal diferencia con un sólido deformable). Un fluido es un conjunto de partículas que se mantienen unidas entre si por fuerzas cohesivas débiles y/o las paredes de un recipiente; el término engloba a los líquidos y los gases (TIPLER, 1986; p. 441). La densidad de una sustancia se define como la relación de la masa y el volumen que ocupa (GIANCOLI, 1988, p. 252):

$$
\begin{aligned}
& \rho\left(\frac{k g}{m^{3}}\right)=\frac{m}{V} \\
& \mathbf{m}=\text { masa en } \mathrm{Kg}, \quad \mathrm{V}=\operatorname{volumen}\left(\mathrm{m}^{3}\right) \text {. }
\end{aligned}
$$

La presión ejercida por un fluido incompresible y en equilibrio dentro de un recipiente de paredes indeformables se transmite con igual intensidad en todas las direcciones y en todos los puntos del fluido.

$$
\begin{gathered}
P(\text { Pascales })=\rho g h+P o \\
\boldsymbol{\rho}=\text { densidad, } \mathbf{g}=\text { gravedad, } \mathbf{h}=\text { altura, } \quad \mathbf{P o}=\text { presión atmosférica. }
\end{gathered}
$$

Los fluidos están sujetos a el principio de Arquímides que afirma que un cuerpo total o parcialmente sumergido en un fluido en reposo, recibe un empuje de abajo hacia arriba igual al RBFTA, v.2, n.2, dezembro de 2015, p. 21 
peso del volumen del fluido que desaloja. Esta fuerza recibe el nombre de empuje hidrostático o de Arquimides (SERWAY y JEWETT, 2005, p.395).

$$
E(N w)=\rho_{f} g V
$$

$$
\rho=\text { densidad del fluido, } g=\text { gravedad, } V=\text { volumen. }
$$

\section{Investigación}

En la investigación, los objetivos planteados fueron, de forma general, describir el fenómeno físico de flotabilidad variable y de forma específica, los objetivos planteados fueron: clasificar las características de los fluidos, analizar el Principio de Arquímides e interpretar el Principio de Pascal. Para aplicar el método, se ha preguntado alguna vez, ¿Como flotan los submarinos? Para dar respuesta a esa pregunta se construirá un divertido juguete que nos permitirá flotar o hundir un objeto (buzo). El juguete consta de una botella plástica vacía (de refresco), un pequeño tubo de ensayo (se puede sustituir por un gotero o un pedazo de pajilla transparente con un extremo sellado) y un pedacito de plastilina. Se le coloca una pequeña cantidad de plastilina alrededor del extremo abierto del tubo se ensayó como contrapeso. El tubo se llena con una parte de agua y otra de aire. La relación aire/agua se determinará experimentalmente de manera que al sumergir el buzo en agua se mantenga críticamente a flote, ver Figura 1:

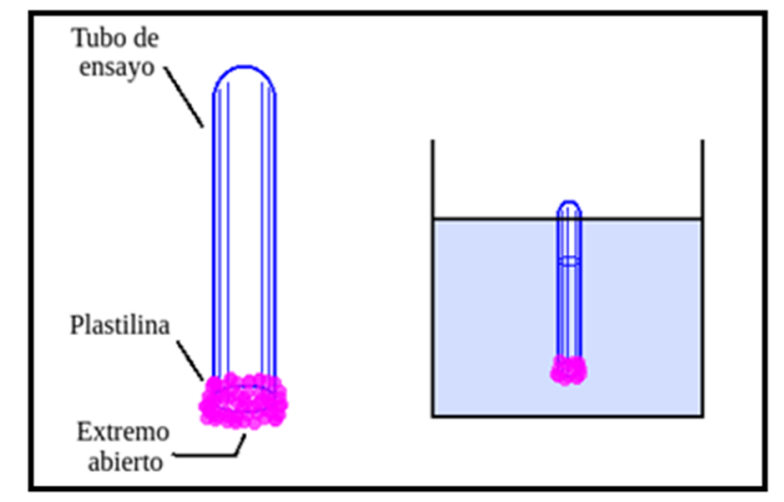

Figura 1: Detalles del buzo y la flotabilidad del mismo.

Fuente: Autor.

La botella se llena totalmente con agua, se introduce el buzo y se cierra herméticamente la botella, como se presenta en la Figura 2. 


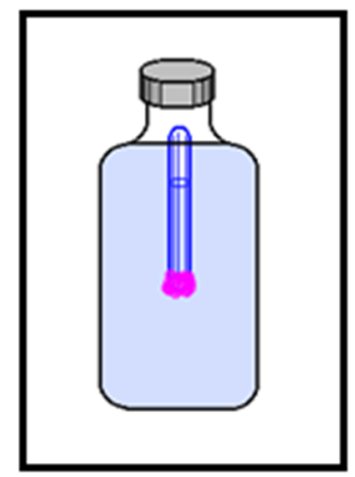

Figura 2: Botella y buzo.

Fuente: Autor.

Sujetamos firmemente la botella con la mano, ver Figura 3, si presionamos la botella el buzo desciende y si liberamos la presión, el buzo asciende!!! ¿Cuál mecanismo físico está actuando en esta experiencia? De manera análoga explicaría la flotabilidad de los submarinos y los peces.

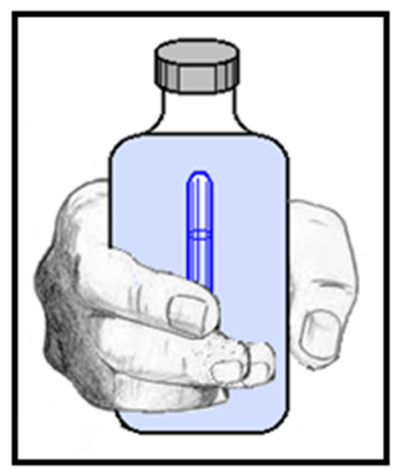

Figura 3: Si se aprieta la botella, el buzo se hunde.

Fuente: Autor.

\section{Procedimientos de las experiencias}

Partiendo de la experiencia central botella/buzo se hace necesario proponer algunas experiencias intermedias para construir y validar los principios físicos relacionados. En cada una de estas experiencias se aplica un interrogatorio que permita medir los conocimientos previos de nuestros estudiantes, en este caso se trata de estudiantes del curso de Física 20, semestre A-2013 de la Facultad de Ingeniería, ULA. Las informaciones que de él se desprende son mostradas en el presente trabajo. Cuando se muestra la experiencia en el aula de clase los estudiantes muestran 
asombro porque con solo apretar y liberar la botella, el buzo se hunde y flota, pero no pueden dar una explicación convincente. Algunos opinan que la presión ejercida por la botella permite la entrada de agua en el buzo, pero no explican el fenómeno como tal. Otros afirman que el buzo contiene la misma cantidad de aire y que no se debería hundir y otros creen que al comprimir el aire éste pesa menos... -el ambiente en el aula de clase es de dudas y confusión por lo que se amerita un estudio detallado y bien fundamentado.

\subsection{Primera experiencia}

Tres objetos de idéntica forma se liberan dentro de un recipiente con agua tal como se muestra en la Figura 4, el primero de ellos flota fácilmente, el segundo flota críticamente y el tercero de ellos se hunde. Las preguntas: ¿Tienen la misma masa? ¿Se hunden o flotan dependiendo de la masa?

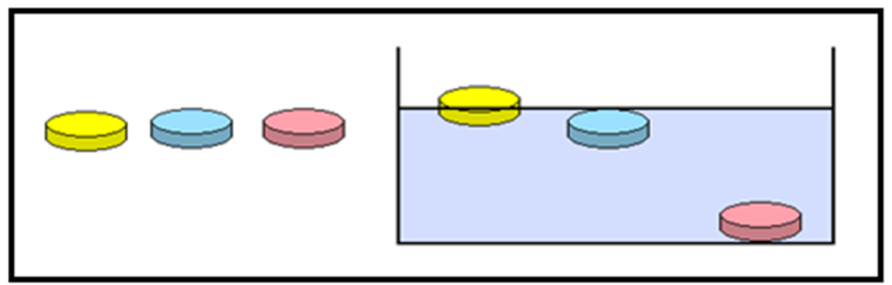

Figura 4: Tres objetos idénticos se ponen a flotar. Fuente: Autor.

A simple vista no podemos diferencias los objetos ya que la forma es la misma. Para hacer referencia a cada uno de ellos se ha pintado con diferentes colores. Se observa que el cuerpo amarillo flota de manera significativa, el cuerpo azul está en el límite de flotación y el cuerpo rojo se ha hundido totalmente.

Al preguntar a la población en estudio, el $100 \%$ ha afirmado que las masas de los cuerpos se relacionan así: amarillo es el liviano, azul medianamente pesado y el rojo es el más pesado y sin duda alguna es totalmente cierto ya que los cuerpos se fabricaron con madera y de manera oculta contienen plomo en su interior. Entonces (por observación) surge de los alumnos la siguiente aseveración: el cuerpo más liviano flota y el más pesado se hunde totalmente.

Los estudiantes experimentaron con diferentes objetos: sacapunta, lápiz, borrador, etc y 
quedaron muy desconcertados ya que unos lápices se hundían y otros flotaban y un voluminoso borrador flotaba a pesar que era mas pesado que el sacapuntas!!!

Es un hecho bien conocido que al colocar un cuerpo liviano sobre el agua éste flota, pero si se coloca un objeto pesado sobre el agua, pues se hundirá irremediablemente, pero, ¿qué tan pesado debe ser un cuerpo para que se hunda o flote sobre el agua?, ¿acaso debemos saber el peso del agua y comparar con el peso del objeto para establecer la flotabilidad del mismo?

\subsection{Segunda experiencia.}

Una botella pequeña que contiene quince canicas se libera en un recipiente con agua y se hunde, si colocamos en el tanque otra botella más grande que la primera y con veinte canicas, obviamente contiene más masa pero, ¿se hunde? Ver Figura 5. Después de haber realizado la experiencia se observa que la botella más pesada y con mayor volumen, no se hunde.

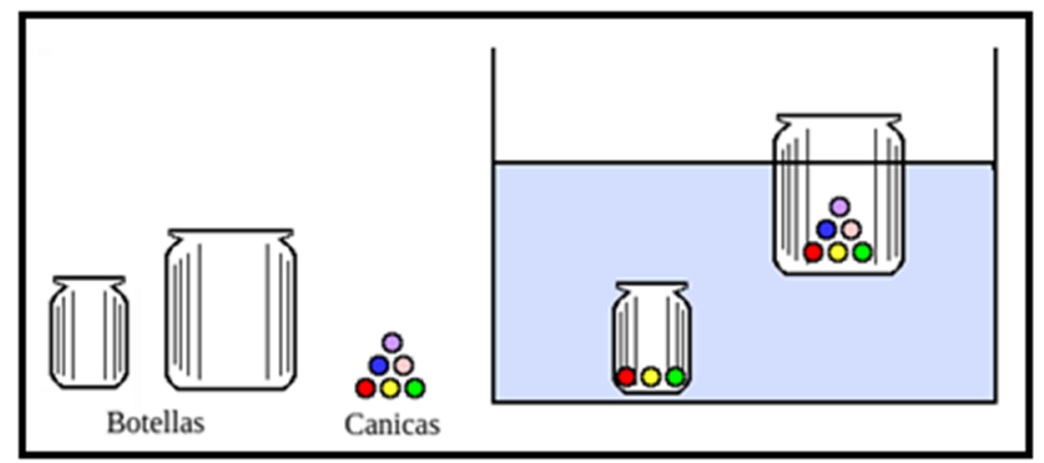

Figura 5: Canicas y botellas en flotación.

Fuente: Autor.

Analice la situación siguiente: un niño se está bañando a la orilla de un gran lago, pone a flotar una pelota de básquet y una canica de vidrio. La pelota de básquet pesa aproximadamente $600 \mathrm{gr}$, la canica pesa 6 gr y el peso del agua que contiene el lago no es determinado pero de seguro pesará algunos miles de kilogramos. Simplemente la pelota flota, la canica se hunde, de manera que el argumento de que los cuerpos pesados se hunden y los livianos flotan no parece ser cierto. Por otro lado el lector puede argumentar que la pelota a pesar de ser más pesada contiene aire en su interior y por eso flota, sin embargo a lo lejos se divisa un buque que contiene una carga muy pesada de varios centenares de toneladas (materiales de construcción) y flota tal 
como lo hace la pelota pero el buque no contiene aire.

Retomemos por un momento el caso de las dos botellas. Una es más pequeña, menos pesada y se hunde y la otra es más grande, más pesada y flota, de manera que una vez más, el argumento los cuerpos pesados se hunden y los livianos flotan no parece ser cierto.

Además del peso, ¿qué otra característica resaltante hay que señalar en el caso de las botellas...? La forma o mejor dicho el volumen. Observe que la botella más grande y pesada es más voluminosa, de manera que la forma de un objeto también contribuye en la determinación de la flotabilidad. La relación entre la masa y volumen se le conoce como la densidad $\boldsymbol{\rho}$. Aproximadamente el 50\% del curso propuso la densidad como causa de la flotabilidad. La masa es directamente proporcional a la densidad, a mayor masa, mayor densidad y a menor masa, menor densidad. La densidad también es inversamente proporcional al volumen, a mayor volumen, menor densidad y a menor volumen, mayor densidad.

Lo anteriormente expuesto nos indicaría el camino para entender el fenómeno de la flotabilidad aunque aún falta un poco más para expresarlo correctamente.

Analicemos la siguiente situación hipotética. Un globo con aire flota en el agua pero no flota sobre el aire; de igual manera una naranja se hunde en el agua pero flota sobre un tanque que contiene un barro espeso, ¿por qué?. Para predecir si un cuerpo flotará debemos saber el medio a través del cual lo vamos a liberar o mejor aún, necesitamos saber la densidad del cuerpo y comparar con la densidad del medio, de manera que si $\boldsymbol{\rho}$ medio es mayor que $\boldsymbol{\rho}$ objeto, el objeto flota. A continuación se anexa una tabla de densidad de algunas sustancias.

Tabla 1: Densidad de sustancias comunes a $0^{\circ} \mathrm{C}$ y $1 \mathrm{~atm}$.

\begin{tabular}{|l|l|l|l|l|l|}
\hline Solidos & $\mathbf{\rho}\left(\mathbf{g r} / \mathbf{c m}^{\mathbf{3}}\right)$ & Líquidos & $\boldsymbol{\rho}\left(\mathbf{g r} / \mathbf{c m}^{\mathbf{3}}\right)$ & Gases & $\boldsymbol{\rho}\left(\mathbf{g r} / \mathbf{c m}^{\mathbf{3}}\right)$ \\
\hline Madera & $0,2-1,0$ & Etanol & 0,79 & Aire & $1,29 \times 10^{-3}$ \\
\hline Hielo & 0,92 & Eter & 0,74 & Amoniaco & $0,77 \times 10^{-3}$ \\
\hline Vidrio & $2,4-6,0$ & Glicerina & 1,26 & Bióxido C & $2,00 \times 10^{-3}$ \\
Acero & 7,8 & Aceite & $0,8-0,95$ & Helio & $0,18 \times 10^{-3}$ \\
\hline Oro & 19,3 & Agua & 1 & Hidrógeno & $0,09 \times 10^{-3}$ \\
\hline Tungsteno & 19,4 & Mercurio & 13,5 & Oxígeno & $1,43 \times 10^{-3}$ \\
\hline
\end{tabular}

Fuente: Giancoli, 1988.

RBFTA, v.2, n.2, dezembro de 2015, p. 26 
Si analizamos el contenido de la tabla, podemos afirmar que el hielo flotará sobre el agua porque $\rho_{\text {hielo }}$ es menor que $\rho_{\text {agua }}$. Algunos estudiantes comentaron que algo similar ocurría cuando intentaban mezclar aceite con agua, el aceite flota sobre el agua dado que $\rho_{\text {aceite }}$ es menor que $\rho_{\text {agua }} \mathrm{y}$ analizaron el caso de la flotabilidad de los globos aerostáticos, pudiendo estos ascender siempre que el contenido del globo sea menos denso que el aire de la atmósfera, por ejemplo aire caliente o gas helio.

\subsection{Tercera experiencia}

Un taco de madera se libera dentro de un recipiente con agua como se muestra en la Figura 6. Responda las siguientes preguntas: ¿Se hunde totalmente?; ¿Flota totalmente?; ¿Flota parcialmente?

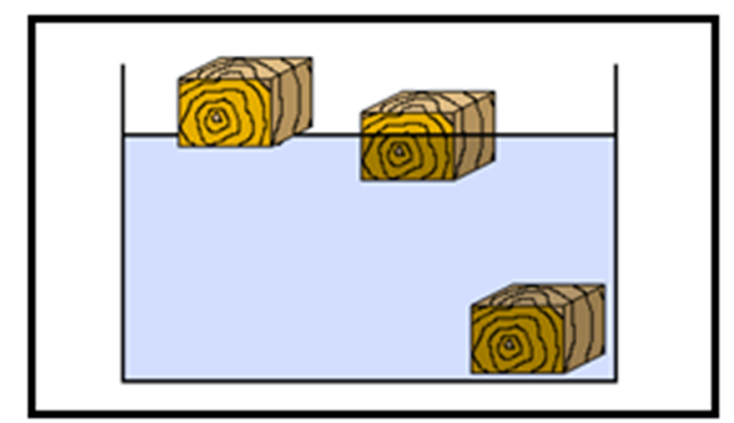

Figura 6: Flotación de un taco de madera. Fuente: Autor

Una vez establecido las condiciones de la flotabilidad (relaciones entre las densidades) podemos preguntar ¿cómo actúa el mecanismo? Si hacemos la experiencia teniendo en cuenta los detalles finos, tendríamos una situación como se ilustra en la Figura 7:

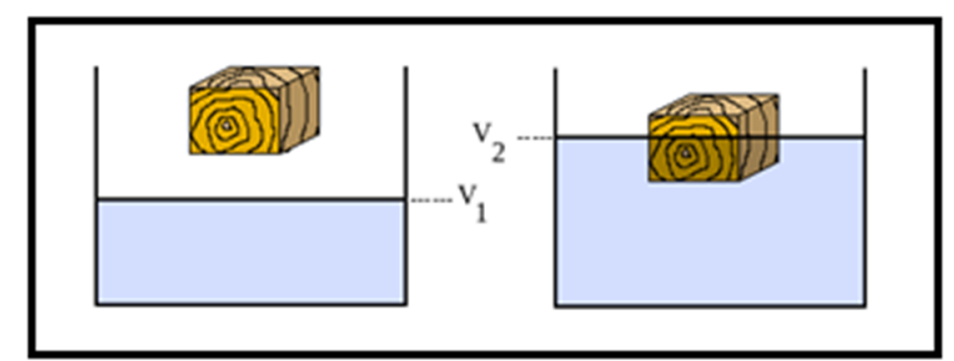

Figura 7: Volumen de agua desplazado por el cuerpo en flotación.

Fuente: Autor. 
El nivel inicial de agua de la cubeta es $\mathrm{V}_{1}$, luego al sumergir el taco de madera el nivel asciende a $\mathrm{V}_{2}$, de manera que el volumen desplazado por la porción del taco que se hunde tiene la variación $\Delta \mathrm{V}=\mathrm{V}_{2}-\mathrm{V}_{1}$. El montaje de la Figura 8 nos permite pesar la porción de taco que se hunde.

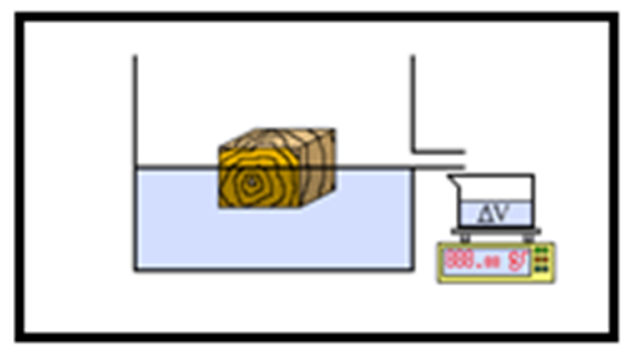

Figura 8: Montaje que permite pesar el volumen de agua desplazado. Fuente: Autor.

Si comparamos el peso $\Delta \mathrm{V}$ del agua desplazada (PAD) con la porción de taco sumergido (PTS), son idénticos, tal como se muestra en la Figura 9:

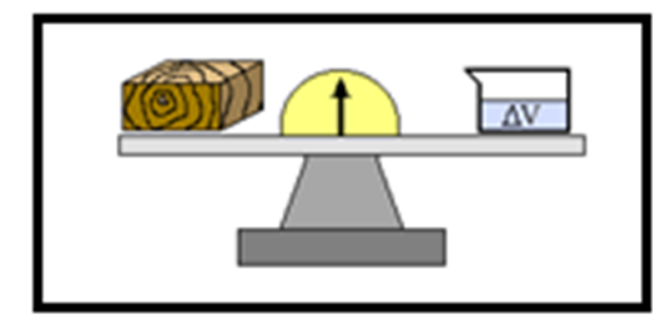

Figura 9: El peso de PAD es igual al peso de PTS. Fuente: Autor.

En condiciones de flotación, la fuerza del peso de PAD se iguala con una fuerza no tan fácil de ver, pero necesaria para anular el peso del PAD - ver Figura 10 -, esa fuerza le llamaremos fuerza de flotación, de igual magnitud pero en sentido contrario que la causa la tensión superficial del agua, tal como lo indica el Principio de Arquímedes. Solo el 10\% del curso tenía una aproximación al Principio de Arquímides. Los estudiantes asociaron el Principio de Arquímides con la experiencia de flotabilidad en la natación, al introducirse en una piscina, notan una subida del nivel del agua y una pérdida de peso aparente del nadador. 


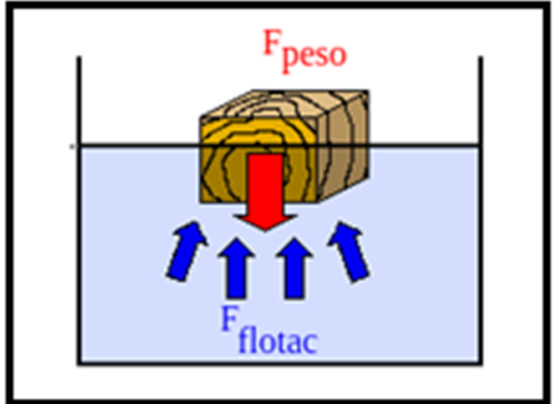

Figura 10: La fuerza de peso se equilibra con la fuerza de flotación.

Fuente: Autor.

\section{Análisis}

Podemos centrar la atención en el hecho de que la flotabilidad de un cuerpo sobre un medio (por ejemplo, cuerpo/líquido) está relacionado con la densidad del cuerpo vs la densidad del medio. Si el cuerpo es más denso que el medio, el cuerpo se hunde dentro del medio.

El hecho que inicia nuestro trabajo se refiere a la flotabilidad o hundimiento del buzo en el agua y podemos determinar tres escenarios posibles en que el buzo: flota sobre la superficie del agua; se hunde y va al fondo del recipiente; se hunde pero lo podemos desplazar en cualquier posición dentro del recipiente.

Analicemos primero el contenido del buzo: recipiente de vidrio, agua, aire y contrapeso de plastilina tal como se muestra en la Figura 1. Sería apropiado designar como densidad promedio del buzo la densidad de las proporciones de los componentes del buzo.

El buzo flotará si su densidad promedio es menor que la densidad del agua, el buzo se hundirá si su densidad promedio es mayor que la densidad del agua y el buzo se mantendrá semi sumergido si su densidad promedio se mantiene cercana a la densidad del agua, pero ¿será acaso que la densidad promedio del buzo no es constante? - en efecto, de eso se trata...!

Dado que la masa de vidrio y plastilina del buzo es constante, se puede variar sutilmente la cantidad de aire y agua para alterar ligeramente la densidad promedio del buzo - ver Figura 11. El mecanismo físico que estaría actuando es el Principio de Pascal.

Si se le aplica una fuerza de compresión a un fluido, éste transmite la fuerza de manera instantánea a través de todo el fluido. Si presionamos exteriormente la botella (con la mano) la 
fuerza se transmitirá de manera instantánea a través del agua, pero ¿cómo afectará esta fuerza al buzo? Recordemos otra propiedad de los fluidos, los gases se comprimen fácilmente y los líquidos son difícil de comprimir, de manera que cuando la fuerza llega al extremo abierto del buzo, ésta comprimirá el aire y permitirá el acceso a mayor cantidad de agua dentro del buzo, aumentando su densidad promedio.

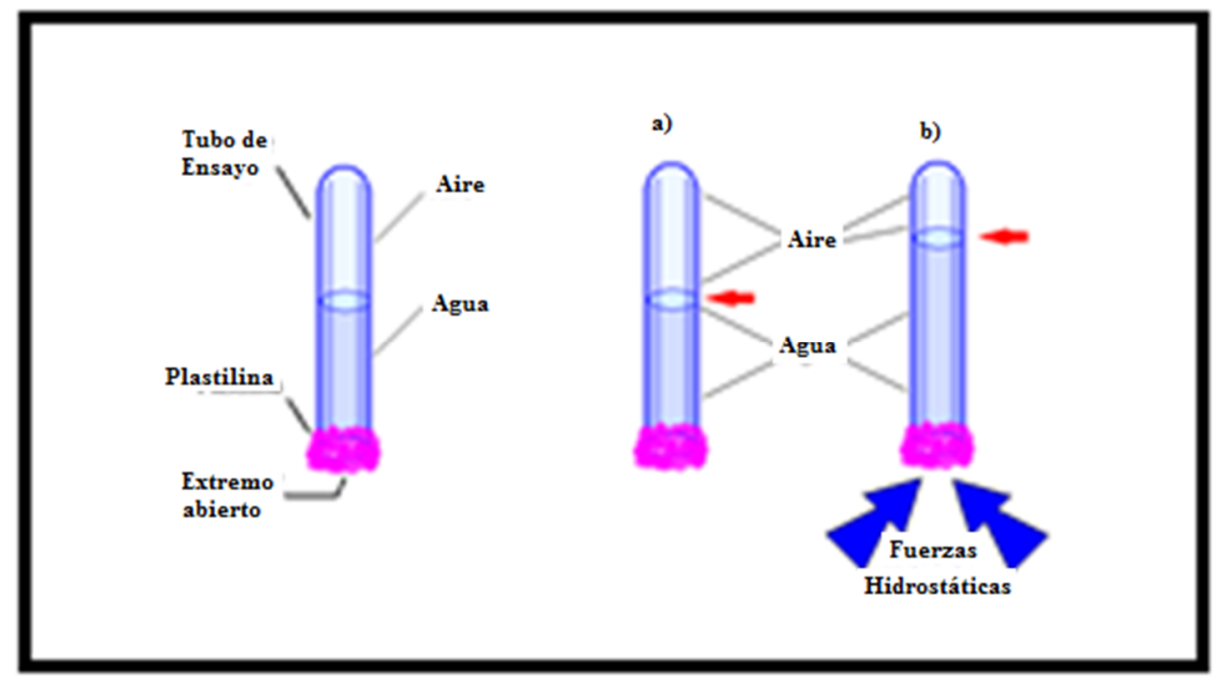

Figura 11: Variación de la relación agua/aire en ausencia o presencia de fuerzas hidrostáticas. a) El buzo se mantiene a flote, note la proporción aire/agua. b) Al aplicar una fuerza hidrostática, el aire se comprime, entra más agua y el buzo se hunde.

Fuente: Autor.

De esta manera podremos presionar suavemente la botella y el buzo descenderá pocos centímetros, al aumentar la presión (o fuerza) el buzo descenderá totalmente pero si dejamos de presionar, el buzo flotará inmediatamente. Lo que anteriormente los estudiantes consideraban contradictorio ahora no lo parece tal, ya que un cuerpo mucho mas pesado que el agua, puede, bajo ciertas situaciones flotar; el conflicto cognitivo ha servido para replantearse los conceptos ya conocidos y lograr incorporar conceptos nuevos para develar el misterio de la flotabilidad variable.

\section{Conclusiones}

El presente trabajo permitió plantear un conflicto cognitivo entre conceptos y situaciones que a primera vista parecían obvias y funcionales pero aplicadas a otras situaciones mostraban 
desacuerdos. Se logró el cambio conceptual que produce en los en los estudiantes una situación contradictoria, este desequilibrio cognitivo condujo a un nuevo conocimiento más amplio y ajustado a la realidad y que seguirá enriqueciéndose en nuevos procesos de aprendizaje; por lo que fue necesario, de manera crítica y formal, rehacer los conceptos de manera significativa, clasificar las características de los fluidos, analizar el Principio de Arquímides y Pascal. Una vez resuelto los desacuerdos, se pudo describir el fenómeno físico de flotabilidad variable, basado en un cambio de densidad del buzo y la relación directa con la densidad del medio en que flota guardando total armonía con los principios y leyes de la hidráulica. Por último, los aportes de este trabajo permiten comprender el mecanismo de flotabilidad de objetos tan pesados como barcos de carga que surcan nuestros mares, naves de inmersión variable como submarinos y peces.

\section{Referencias}

GIANCOLI, D.: Física General. México, ed. Prentice Hall, México 1988.

LEA, S.M.; BURKE, J.R.: Física: La naturaleza de las cosas, vol 1, ed. International Thomson, México 1999.

PIAGET, J.: L'équilibration des structures cognitives. Probléme central da développement. Presses Universitaires de France, Paris, 1975.

SERWAY, A.; JEWETT, J.: Fisica para Ciencias e Ingenierías, ed.Thomson, México, 2005.

TIPPLER P.: Física. Reverté, España, 1986.

\section{Informaciones adicionales de otros autores:}

María José Torres Fernández: Licenciatura en Ciencias Físicas en la Universidad de los Andes, Venezuela, e-mail: mariajosetorresastronomy@hotmail.com

Adriana Carolina Vasquez Ramirez: Licenciatura en Ciencias Físicas en la Universidad de los Andes, Venezuela, e-mail: adn999_666@hotmail.com

Artigo recebido para avaliação em 05/05/2015 e aceito para publicação em 17/12/2015. 